\title{
Increase in Size of Flaked Particles Suddenly Generated by Impulsive Force in Mass-production Plasma Etching Equipment
}

\author{
Yuji KASASHIMA*1 and Fumihiko UESUGI*1 \\ ${ }^{*}$ Advanced Manufacturing Research Institute, National Institute of Advanced Industrial Science and Technology (AIST), \\ 807-1 Shukumachi, Tosu-shi, Saga, 841-0052 Japan
}

(Received July 26, 2016, Accepted September 12, 2016)

We investigate flaked particles instantaneously generated in mass-production plasma etching equipment. Particles flaking off from films deposited on the ground electrode are detected by using laser light scattering system. Not only the number of particles but also the size of them obviously increases when micro-arc discharge occurs around a wafer and floating potential (inner wall potential) changes instantaneously. The results of this study provide evidence that electric field stress acts as impulsive force due to rapidly changing floating potential, causing dramatically breaking of deposited film and many large particles.

\section{Introduction}

In the mass production of large scale integrated circuit (LSI), particle contamination in plasma etching equipment causes serious damage. This is because the particles can short-circuit LSI and significantly decrease production yields ${ }^{1-10)}$. The mass-production equipment must be stopped for cleaning of the etching chambers and maintenance, hence overall equipment efficiency (OEE) also decreases. Therefore, development of particle-free processes and equipment is highly important to improve the production yield and OEE, and to reduce the production cost.

During plasma etching process, the etching reaction products adhere to the inner walls of the process chamber, and gradually deposit as films as many wafers are processed. The particles are generated by flaking of the deposited films caused by electric field stress that acts at the boundary between the insulating inner wall and the film ${ }^{6,11-15)}$. The electric field is formed between the inner wall surface at the floating potential (inner wall potential) and the chamber at the ground potential. The generation of particles depends on the number of processed wafers; the thicker the films deposited on the inner walls, the more particles are generated. In addition, a lot of particles sometimes suddenly flake off and give rise to many defective LSI devices. We have previously elucidated the mechanism that the number of particles instantaneously increases when the electric field stress acts as impulsive force ${ }^{11-14)}$. An impulsive force is known in classical mechanics as a force becoming much larger as the time to act becomes much shorter ${ }^{16,17)}$. We have investigated the mechanism of sudden generation of many flaked particles, and elucidated that many particles are generated when the electric field stress changes instantaneously and works as an impulsive force ${ }^{11}$. We have also clarified that the deposited film can largely deform and flake off as many particles owing to the impulsive force of electric filed stress, from the calculated result using the forced oscillation model ${ }^{12)}$. In addition, we have elucidated that micro-arc discharge is a cause of the sudden generation of many flaked particles ${ }^{13)}$. The existence of the phenomenon of the impulsive force of electric field stress has been confirmed by using the standard particles $^{14)}$. In these previous studies, the impulsive force is brought about by the rapid change in the inner wall potential when a wafer unusually moves owing to insufficient wafer chucking force and micro-arc discharge occur. In mass-production line, not only the number of particles but also the size of them is a critical factor, because particularly the production yield depends on both the number and the size of particles. Under the condition that the impulsive force works, however, characteristic of the size of particle has not been elucidated. In this study, therefore, we investigate the size distribution of flaked particles in the case that micro-arc discharge occurs and the impulsive force acts. The results indicate that, in addition to the increase in the number of particles, the size of them clearly increases owing to large scale breaking of deposited films caused by the impulsive force.

\section{Experimental methods}

The experimental apparatus shown in Fig. 1 is the mass-production reactive ion etching (RIE) equipment with a capacitive $\mathrm{rf}$ discharge $(13.56 \mathrm{MHz})^{11)}$. A wafer of diameter $200 \mathrm{~mm}$ is chucked on the powered electrode by

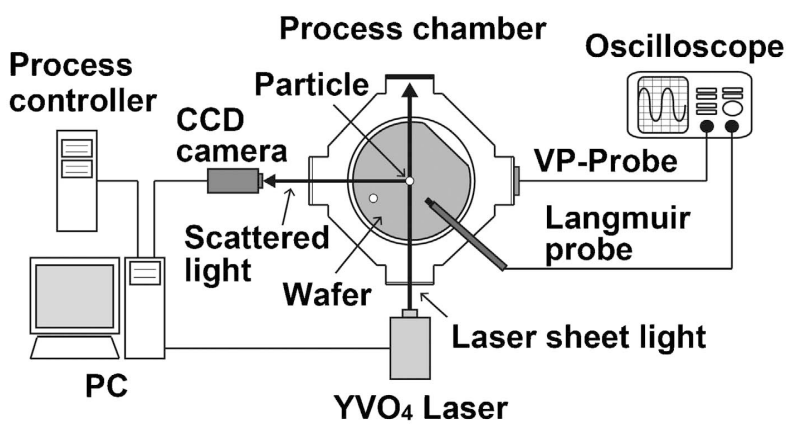

Fig. 1 Schematic view of the experimental setup. 
an electrostatic chuck (ESC) with backside cooling by $\mathrm{He}$ gas. The etching process sequence and equipment parameters used in this study are similar to those used in manufacturing facilities. A titanium etching process is used because the process often causes serious particle contamination. The etching gas consists of $\mathrm{SF}_{6}$ with a flow rate of $100 \mathrm{sccm}$ and $\mathrm{O}_{2}$ with a flow rate of $6 \mathrm{sccm}$ at a pressure of $18 \mathrm{~Pa}$. The distance between the ground and powered electrodes is $60 \mathrm{~mm}$. An rf power of 1000 $\mathrm{W}$ is supplied and the etching time is set at $15 \mathrm{~s}$.

The in-situ particle monitoring system and viewing port style plasma probe (VP-Probe) used in this study are described in detail in Ref. 11. In the particle monitoring system, a sheet-like laser beam is introduced in a plane parallel to a wafer in the processing chamber at a distance of $4 \mathrm{~mm}$ from the ground electrode (upper electrode). An image-intensified charge-coupled device (CCD) camera detects light scattered by particles crossing the beam. Changes occurring during a $0.1 \mathrm{~s}$ period are recorded in one CCD camera image, and images are captured approximately every $0.6 \mathrm{~s}$. The system can detect particles together with the working signal of the RIE equipment. The VP-Probe can detect transient changes in floating potential on the insulated inner surface of the chamber wall caused by micro-arc discharge ${ }^{18)}$. Signals from the VP-Probe are monitored simultaneously with the working signals of rf power and CCD camera using a digital oscilloscope, with a sampling frequency of 1 MHz. To investigate the change in the inner wall potential, the floating potential is measured by using a Langmuir probe. The potential is related to the floating potential formed on the chamber inner wall ${ }^{19}$. The probe electrode (tungsten wire) has a radius of $0.6 \mathrm{~mm}$ and a length of $3.5 \mathrm{~mm}$, and is set at a position of approximately $40 \mathrm{~mm}$ above the wafer and approximately $10 \mathrm{~mm}$ distant from the center of the wafer. Langmuir probe signals are monitored by using the digital oscilloscope, with a sampling frequency of $50 \mathrm{MHz}$.

\section{Results and discussion}

In the experiment, micro-arc discharge is induced by applying a little moisture to the wafer surface before the wafer is carried to the process chamber. This simulates the arcing caused by residual moisture in the process chambers in mass-production line, which sometimes occurs after wet cleaning process. The arcing occurring around a wafer is detected by the VP-Probe and visually observed. Figure 2 is typical image of flaked particles detected when micro-arc discharge occurs. Although the arcing occurs at the wafer, these particles are generated from the ground electrode; that is, these simultaneously occur at the different places. Figure $\mathbf{3}$ shows typical signal of the change in the floating potential $V_{\mathrm{f}}$ measured by Langmuir probe. The floating potential markedly and rapidly changes within microseconds order owing to micro-arc discharge, which occurs approximately at $2 \mu \mathrm{s}$. After the arcing occurs, the probe signal decreases and then increases. Electrons originating from the micro-arc discharge would increase instantaneously, so that the floating potential decreases. This is because when the

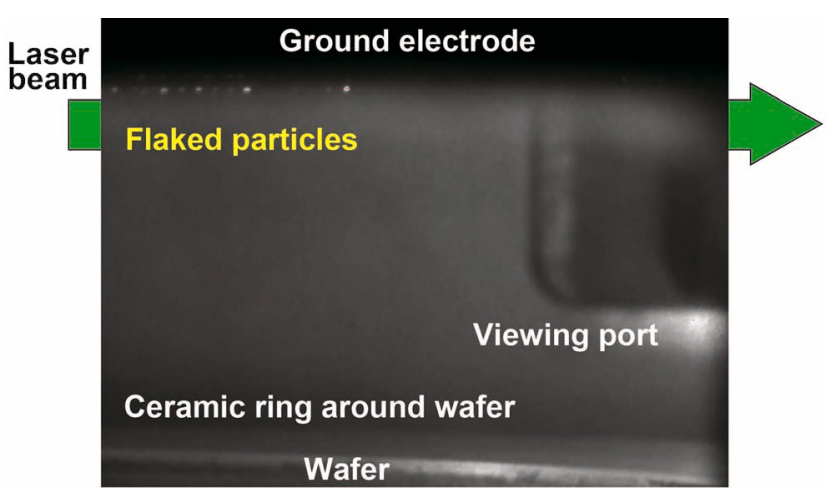

Fig. 2 (Color online) Typical image of many flaked particles when micro-arc discharge occurs.

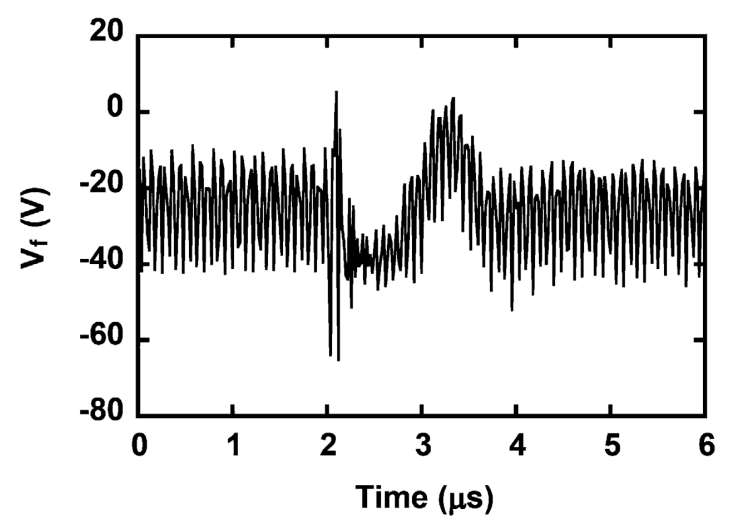

Fig. 3 Signal of the floating potential $V_{\mathrm{f}}$ measured by using Langmuir probe when micro-arc discharge occurs.

electron instantaneously increases due to the arcing, the potential of plasma $V_{\mathrm{s}}$ would decrease and the electron saturation current $J_{\text {es }}$ can increase, and as a result the floating potential $V_{\mathrm{f}}$ can decrease $\left(V_{\mathrm{f}} \propto V_{\mathrm{s}},-J_{\mathrm{es}}\right)^{19)}$. Then, the arcing can cause sudden impedance mismatching and weaken the rf power, hence the potential changes to positive direction. The decrease in the amplitude of the probe signal, whose frequency is $13.56 \mathrm{MHz}$, shown approximately from 2 to $3 \mu$ s would also be caused by the weaken the rf power. As the arcing is extinguished and the rf power returns, the probe signal then returns to the initial value before the arcing. As a consequence, these results indicate that the inner wall potential changing rapidly causes the electric field stress which can work as impulsive force and many flaked particles ${ }^{11-14)}$.

In the experiment to investigate the relationship between the impulsive force effect and the number and the size distribution of flaked particles, fifteen wafers are processed. Within the etching processes, micro-arc discharge is observed in the process of five wafers. Figure 4 shows the relation between the number of particles per wafer and the intensity of scattered light by the particles. An intensity of 4095 is the upper detection limit of the CCD camera of the particle monitoring system. The system detects particles on the principle of Mie scattering; thus, scattering intensity is not necessarily directly proportional to particle size ${ }^{20,21)}$. However, based on Mie 


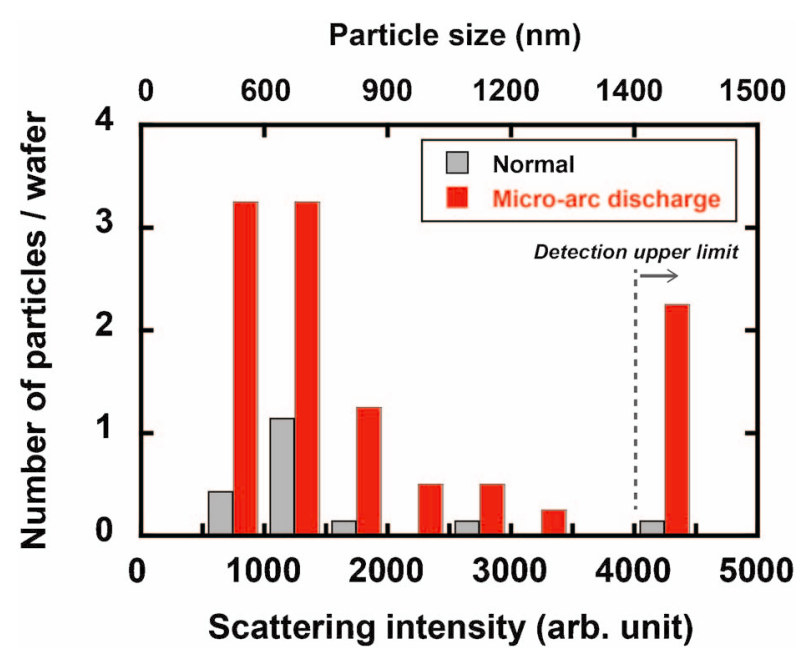

Fig. 4 (Color online) Relationship between the number of particles per wafer and intensity of laser scattering light, and particle size (diameter). An intensity of 4095 is the upper detection limit of the CCD camera of the particle monitoring system. Normal: wafer is normally processed. Micro-arc discharge: micro-arc discharge occurs during etching.

scattering, scattering intensity tends to increase with particle size and the size can be roughly estimated. The tendency is not limited to a spherical particle but scattering intensity can be relatively easy to calculate in the case of spherical particle. Hence the diameter of particle is estimated by using the intensity of scattered light by particle and the intensity calculated by theory of Mie scattering by a spherical particle. The estimated values are indicated at the upper horizontal axis. Compared to the case that wafer is normally processed (shown as Normal), in the case that micro-arc discharge occurs, the number of particles clearly increases in each diameter range. In addition, a noteworthy fact is that the particles with larger diameter are generated when the arcing occurs, whereas such larger particles are few when the arcing does not occur. In other words, the result provides evidence that the electric field stress acts as an impulsive force due to the rapid change in the floating potential caused by microarc discharge, and as a result the deposited film is severely deformed and flakes off as large and many particles. The existence of the impulsive force is demonstrated. The deposited film composed of etching reaction products is polycrystalline, hence when the film is largely and rapidly deformed by the impulsive force, the grain boundaries of the films would be easily cracked. As a consequence, the grains flake off and particles with large size are generated. The further detailed mechanism of the breaking of the deposited film will be investigated in future. Figure 5 shows another image that detects the impulsive force effect. The film deposited on the ground electrode is dramatically detached and numerous particles are flaking off.

\section{Conclusions}

Flaked particles suddenly generated in mass-production plasma etching are investigated. When rapid changes of floating potential (inner wall potential) are

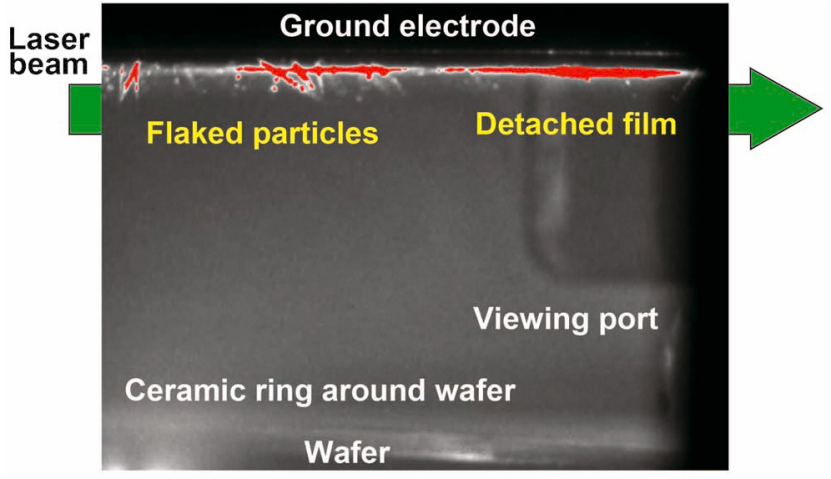

Fig. 5 (Color online) Typical image of dramatically flaking of deposited film owing to impulsive force.

caused by micro-arc discharge, the number of particles and the size of them clearly increase. The rapid change in the floating potential and the electric field causes large increase in electric field stress. This is due to the stress can act as an impulsive force when the electric field changes instantaneously. The results of this study demonstrate the existence of the impulsive force owing to instantaneous changing floating potential, which causes largely breaking of deposited film and many large particles. To prevent such sudden generation of many flaked particles, to suppress the instantaneous changes in the floating potential caused by anomalies such as micro-arc discharge and unusual wafer movement is highly important.

\section{Acknowledgement}

This work was partially supported by JSPS KAKENHI Grant Number 16K21676. The authors thank Professor Akira Ando, Dr. Kazunori Takahashi, Professor Toshiro Kaneko, and Professor Katsuyoshi Washio (Tohoku University); Dr. Taisei Motomura, Dr. Tatsuo Tabaru, and Ms. Natsuko Nabeoka (AIST) for their assistance and helpful discussions.

\section{References}

1) N. Ito, T. Moriya, F. Uesugi, M. Matsumoto, S. Liu and Y. Kitayama: Jpn. J. Appl. Phys., 47 (2008) 3630.

2) F. Uesugi, N. Ito, T. Moriya, H. Doi, S. Sakamoto and Y. Hayashi: J. Vac. Sci. Technol. A, 16 (1998) 1189.

3) N. Ito, T. Moriya, F. Uesugi, H. Doi, S. Sakamoto and Y. Hayashi: J. Vac. Sci. Technol. B, 16 (1998) 3339.

4) T. Moriya, N. Ito, F. Uesugi, Y. Hayashi and K. Okamura: J. Vac. Sci. Technol. A, 18 (2000) 1282.

5) T. Moriya, N. Ito and F. Uesugi: J. Vac. Sci. Technol. B, 22 (2004) 2359.

6) T. Moriya, H. Nakayama, H. Nagaike, Y. Kobayashi, M. Shimada and K. Okuyama: IEEE Trans. Semicond. Manuf., 18 (2005) 477.

7) H.-S. Jun: Jpn. J. Appl. Phys., 52 (2013) 066203.

8) G. Lapenta and J. U. Brackbill: Plasma Sources Sci. Technol., 6 (1997) 61.

9) S. J. Choi, P. L. G. Ventzek, R. J. Hoekstra and M. J. Kushner: Plasma Sources Sci. Technol., 3 (1994) 418.

10) M. A. Hussein and R. B. Turkot, Jr.: IEEE Trans. Semicond. Manuf., 19 (2006) 146.

11) Y. Kasashima, N. Nabeoka and F. Uesugi: Jpn. J. Appl. Phys., 52 (2013) 066201. 
12) Y. Kasashima, N. Nabeoka, T. Motomura and F. Uesugi: Jpn. J. Appl. Phys., 53 (2014) 040301.

13) Y. Kasashima, T. Motomura, N. Nabeoka and F. Uesugi: Jpn. J. Appl. Phys., 54 (2015) 01AE02.

14) Y. Kasashima and F. Uesugi: Jpn. J. Appl. Phys., 53 (2014) 110308.

15) J. A. Stratton: Electromagnetic Theory (McGraw-Hill, New York, 1941) Chap. 2, p. 83.

16) L. D. Landau and E. M. Lifshitz: Course of Theoretical Physics (Elsevier, Amsterdam, 1976) 3rd ed., Vol. 1, Chap. 5, p. 58.

17) H. Goldstein, C. Poole and J. Safko: Classical Mechanics (Ad-
dison-Wesley, Reading, MA, 2001) 3rd ed., Chap. 2, p. 34.

18) M. Yasaka, M. Takeshita and R. Miyagawa: Jpn. J. Appl. Phys., 42 (2003) L157.

19) M. A. Lieberman and A. J. Lichtenberg: Principles of Plasma Discharges and Materials Processing (Wiley, New York, 2005) 2nd ed., Chap. 6, p. 165.

20) H. C. van de Hulst: Light Scattering by Small Particles (Dover, New York, 1981) Chap. 9, p. 114.

21) F. Bohren and D. R. Huffman, Absorption and Scattering of Light by Small Particles (Wiley, New York, 1983) Chap. 4, p. 83. 